\title{
Vibrio splendidus biovar II as the causative agent of bacillary necrosis of Japanese oyster Crassostrea gigas larvae
}

\author{
G. Sugumar ${ }^{1,2}$, T. Nakai ${ }^{2, *}$, Y. Hirata ${ }^{3}$, D. Matsubara ${ }^{3}$, K. Muroga ${ }^{2}$ \\ ${ }^{1}$ Fisheries College and Research Institute, Tamil Nadu Veterinary and Animal Sciences University, Tuticorin 628008, India \\ ${ }^{2}$ Faculty of Applied Biological Science, Hiroshima University, Higashihiroshima 739-8528, Japan \\ ${ }^{3}$ Hiroshima Prefectural Farming Fisheries Association, Takehara 729-2313, Japan
}

\begin{abstract}
Recurrent outbreaks of a disease leading to mass mortalities in an oyster (Crassostrea gigas) hatchery located in western Japan were investigated. The disease occurred regularly in 2 - to 8-d-old larvae and has been experimentally controlled in the hatchery by treating the larval rearing water with streptomycin, without ascertaining the etiological agent. The signs of the disease and the course of infection resembled bacillary necrosis reported in oysters and other bivalve molluscs in the USA and Europe. Quantitative and qualitative examinations of the bacterial flora of hatchery samples including source water, broodstock, larval feed and larvae revealed a very high total bacterial load and presumptive vibrios in diseased larvae. Further, the bacterial profile revealed that Vibrio spp. constituted approximately 60 to $95 \%$ of the bacteria isolated from infected larvae and most isolates were identified as $V$. splendidus biovar li and $V$. harveyi, suggesting their possible role in the disease. However, experimental challenges proved the pathogenicity of $V$. splendidus II Several isolates of $V$. splendidus II from infected larvae were highly pathogenic, producing $100 \%$ mortality at levels of $10^{5} \mathrm{cfu} \mathrm{m}^{-1}$ in $24 \mathrm{~h}$, while isolates from other sources demonstrated a low degree of virulence. Detection of $V$. splendidus Il from broodstock, especially in the gonad of a few breeders, suggests the probability that broodstock could be the source and route of transmission of this pathogen.
\end{abstract}

KEY WORDS: Bacillary necrosis Vibriosis - Crassostrea gigas - Larvae - Vibrio splendidus II Pathogenicity

\section{INTRODUCTION}

Bacillary necrosis, a disease of larval and juvenile bivalve molluscs, is one of the major problems in hatcheries, causing serious production losses. Marine vibrios have often been incriminated in such mortalities and the disease, also termed larval vibriosis, has been reported from several geographical locations, especially in the USA and Europe (Elston 1984). Bacillary necrosis of several species of larval bivalves was first described to be caused by Gram-negative motile rods, some of which were subsequently identified as Vibrio alginolyticus and $V$. anguillarum (Tubiash et al. 1965,1970 ). Involvement of $V$. anguillarum in larval

- Addressee for correspondence.

E-mail: nakaitt@ipc.hiroshima-u.ac.jp mortalities of oyster Ostrea edulis (DiSalvo et al. 1978) and Vibrio spp. in epizootic of Crassostrea virginica (Brown \& Losee 1978, Elston et al. 1981) were reported in the USA. Jeffries (1982) isolated 3 different Vibrio species from diseased $C$. gigas larvae in the United Kingdom and experimentally found that strains of $V$. anguillarum were highly pathogenic, while $V$. alginolyticus and $V$. splendidus were, respectively, moderately and less pathogenic to oyster larvae. However, the isolates reported as $V$. anguillarum by Tubiash et al. (1970) and Jeffries (1982) were later classified as a new species under the name $V$. tubiashii (Hada et al. 1984). Recently, a $V$. anguillarum-related strain causing epizootics of scallop Argopecten purpuratus larvae was reported in Chile (Riquelme et al. 1995a) and V. splendidus was among the isolates from diseased scallop Pecten maximus larvae in France (Nicolas et al. 1996). Although bivalve larval vibriosis implicating 
these Vibrio species has been reported from diverse geographical locations, it is regarded only as an opportunistic disease occurring in crowded husbandry systems (Elston 1984, 1993).

The Japanese or Pacific oyster Crassostrea gigas has been intensively cultured along the Pacific coast of Japan since the 19th century and has depended fully on natural spats. In 1993, mass production of triploid larvae of $C$. gigas by the application of thermal shock and caffeine treatment to fertilized eggs (Akashige \& Kusuki 1996) was initiated at the hatchery of the Hiroshima Prefectural Farming Fisheries Association (HPFFA) in western Japan along the Seto Inland Sea. However, there were always high mortalities, especially in early stages ( 2 to $8 \mathrm{~d}$ ) of larval development, which serrousiy limited production. Experinenidal treatment of rearing water with antibiotic $\left(7.2 \mu \mathrm{g} \mathrm{ml} \mathrm{m}^{-1}\right.$ streptomycin), under the presumption that the mortality could be due to bacterial infection, controlled the disease, leading to successful rearing of larvae in the hatchery since 1995. Against this background, a bacteriological investigation was conducted at the HPFFA hatchery to determine the causative agent(s) of the disease and the source of infection.

\section{MATERIALS AND METHODS}

Sampling. Two batches of triploid larvae produced in the hatchery during May and June 1997 were used for the study to determine the causative agent of the disease. The experimental strategy included bacteriological examinations of cultured larvae in experimental tanks (500 l) and in regular production tanks $\left(20 \mathrm{~m}^{3}\right)$ with and without streptomycin treatment of rearing water as described below: One batch of larvae was reared at a density of 4 larvae $\mathrm{ml}^{-1}$ in 2 experimental tanks and another batch of larvae was reared in the regular production tanks at a density of 2 larvae $\mathrm{ml}^{-1}$. Both the batches were reared in $1 \mu \mathrm{m}$-filtered seawater at $25 \pm 1^{\circ} \mathrm{C}$ as routinely performed in the hatchery. A concentration of $7.2 \mu \mathrm{g}$ (concentration of active ingredient) $\mathrm{ml}^{-1}$ of streptomycin was maintained in larval rearing water in 1 of the 2 experimental and 1 of the 2 regular production tanks while the other 2 served as controls. Streptomycin level was maintained by replacing approximately $50 \%$ of tank water with freshly medicated water every 2 or $3 \mathrm{~d}$ and a $100 \%$ change during mid-cycle of larval production. Samples of larvae and water were drawn from both the treated and untreated tanks with the first sign of disease indicated by visible aggregation of larvae on tank bottom. In addition, samples of raw seawater, filtered seawater, larval feed (Chaetoceros calcitrans and Pavlova lutheri), broodstock and breeder tank water were also analysed bacteriologically. The shell length of larvae was determined by measuring a sample of 50 larvae using a Nikon profile projector, and larval survival was estimated by counting the larvae in sample volumes from each tank at the beginning and end of the production cycle (18 d).

Bacteriology. Inactive larvae, which settled to the bottom of the tanks with the onset of the disease, were siphoned out and filtered through a plankton net of $50 \mu \mathrm{m}$ mesh size. Healthy larvae, which were swimming in the water column of the tanks, were collected by filtering water through a plankton net. Larval samples were washed with $0.2 \mu \mathrm{m}$-filtered seawater and resuspended in $2 \mathrm{ml}$ of sterile seawater, and the larvae in $100 \mu$ l of the suspension were counted using a Neubauer chamber under a low power $(60 x)$ microscope. Homogenates of larvae, algae and adult oysters and samples of water were serially diluted, plated on Marine Agar 2216 (MA, Difco) and Thiosulfate Citrate Bile salt Sucrose (TCBS) agar (Difco) and incubated at $25^{\circ} \mathrm{C}$. Colonies on TCBS and MA were counted after 48 and $72 \mathrm{~h}$, respectively. Gonads of broodstock were sampled using an inoculation wire loop after making an incision in the gonad with a sterile scalpel, and were streaked directly on TCBS agar plates. For qualitative analysis, colonies on MA plates were picked randomly, purified and subjected to a battery of standard biochemical tests that identified them to generic level following the scheme described by the Oceanographical Society of Japan (1990). Presumptive vibrios including all representative morphotypes on TCBS and those on MA confirmed as Vibrio species were further assigned to various species according to Alsina \& Blanch (1994a, b).

Drug sensitivity. Drug sensitivity of isolates of Vibrio splendidus II was assayed by the disc diffusion method (Barry \& Thornsberry 1991) on Mueller-Hinton agar (Eiken Co., Ltd, Tokyo) supplemented with $1.5 \%$ $\mathrm{NaCl}$, against the following 15 antibiotics and chemotherapeutics (in $\mu \mathrm{g}$ per disc): streptomycin (50), kanamycin (50), vancomycin (5), ampicillin (30), oxycillin (30), colistin (150), oxytetracycline (30), sulfactumcefaperazolin (30), cefaperazolin (30), cephalexin (30), cefazolin (30), erythromycin (30), novobiocin (20), nalidixic acid (50) and chloramphenicol (100). Depending upon the zone of inhibition, as per manufacturer's guidelines, the isolates were classified as sensitive (S), weakly sensitive (WS) and resistant (R).

Pathogenicity assays. Healthy oyster larvae were challenged with strains of dominant Vibrio species to determine their pathogenicity. A total of 23 isolates consisting of strains of Vibrio splendidus II, V. harveyi, $V$. alginolyticus and a $V$. anguillarum-like organism were tested. Static cultures of bacteria grown in Marine Broth 2216 (Difco) at $25^{\circ} \mathrm{C}$ for $24 \mathrm{~h}$ were cen- 
trifuged at $3000 \times g$ for $20 \mathrm{~min}$, washed twice and resuspended in phosphate-buffered saline (PBS) (1.5\% $\mathrm{NaCl}$. Ten-fold dilutions of the cell suspensions were prepared and approximately $10^{3}$ to $10^{7}$ colony-forming units (cfu) $\mathrm{ml}^{-1}$ were added to 5-to 8-d-old larvae (shell length $=107.8 \pm 12.1$ to $133.4 \pm 18.2 \mu \mathrm{m}$ ) maintained at a density of 4 larvae $\mathrm{ml}^{-1}$ in $10 \mathrm{ml}$ of filter sterilized seawater in 6-well cell culture chambers (Falcon). Chambers without addition of bacteria served as controls. These assays were performed in duplicates at $25^{\circ} \mathrm{C}$ and larval survival was recorded after $24 \mathrm{~h}$. Larvae sinking to the bottom of the chamber with no apparent intravalvular movement as observed by a low power microscope were considered dead. Based on larval mortality at the end of $24 \mathrm{~h}$ exposure, the $50 \%$ lethal dose $\left(\mathrm{LD}_{50}\right)$ was determined in all possible cases by the method of Reed \& Meunch (1938). Reisolation of the introduced bacteria in experimentally infected larvae was also performed. Moribund/dead larvae from challenged wells and larvae from control wells were carefully picked up with a pasteur pipette, washed in sterile seawater and the whole larvae directly plated on TCBS agar. Colonies that developed were characterized biochemically as described earlier.

Table 1. Quantitative bacterial analysis of samples from experimental tanks

\begin{tabular}{|c|c|c|c|c|}
\hline Sample & $\begin{array}{l}\text { Total viable } \\
\left(\mathrm{cfu} \mathrm{g}^{-1} \text { or } \mathrm{ml}^{-1}\right)\end{array}$ & $\begin{array}{l}\text { e count } \\
\text { (cfu larva-1) }\end{array}$ & $\begin{array}{l}\text { Presumptive vit } \\
\left(\mathrm{cfu}^{-1} \text { or } \mathrm{m}^{-1} \text { ) }\right.\end{array}$ & $\begin{array}{l}\text { ibrio count" } \\
\text { (cfu larva ) }\end{array}$ \\
\hline \multicolumn{5}{|l|}{ Untreated tank } \\
\hline Inactive larvae & $1.4 \times 10^{11}$ & $1.1 \times 10^{5}$ & $1.9 \times 10^{9}(79)$ & $1.5 \times 10^{3}$ \\
\hline Active larvae & $4.9 \times 10^{8}$ & $3.9 \times 10^{2}$ & $2.1 \times 10^{6}(54)$ & $1.7 \times 10^{0}$ \\
\hline Tank water & $8.3 \times 10^{4}$ & & $6.0 \times 10^{1}(100)$ & \\
\hline \multicolumn{5}{|c|}{ Streptomycin-treated tank } \\
\hline Inactive larvae & $8.4 \times 10^{8}$ & $6.6 \times 10^{2}$ & $3.7 \times 10^{7}(72)$ & $2.9 \times 10^{1}$ \\
\hline Active larvae & $5.8 \times 10^{4}$ & $4.6 \times 10^{3}$ & $4.5 \times 10^{h}(23)$ & $3.5 \times 10^{6}$ \\
\hline Tank water & $1.5 \times 10^{5}$ & & $<1.0 \times 10^{1}$ & \\
\hline Raw seawater & $1.5 \times 10^{3}$ & & $<1.0 \times 10^{1}$ & \\
\hline \multicolumn{5}{|c|}{ a Values in parentheses are \% of sucrose-negative vibrios } \\
\hline
\end{tabular}

Table 2. Vibrio species associated with samples from experimental tanks. $\mathrm{n}=$ number of isolates picked for species characterization

\begin{tabular}{|c|c|c|c|c|c|}
\hline \multirow[t]{2}{*}{ Species } & \multicolumn{3}{|c|}{ Untreated tank } & \multicolumn{2}{|c|}{ Streptomycin-treated tank } \\
\hline & $\begin{array}{c}\text { Inactive } \\
\text { larvae } \\
(\mathrm{n}=20)\end{array}$ & $\begin{array}{l}\text { Active } \\
\text { larvae } \\
(\mathrm{n}=8)\end{array}$ & $\begin{array}{c}\text { Tank } \\
\text { water } \\
(\mathrm{n}=7)\end{array}$ & $\begin{array}{l}\text { Inactive } \\
\text { larvae } \\
(n=17)\end{array}$ & $\begin{array}{l}\text { Active } \\
\text { larvae } \\
(n=5)\end{array}$ \\
\hline V. aestuarinus & 1 & - & - & - & - \\
\hline$V$. anguillarum-like & e 1 & - & - & - & 1 \\
\hline V. harveyi & 4 & - & 1 & - & - \\
\hline V. mediterranei & - & 3 & - & 4 & 3 \\
\hline Vibrio Phenon 14 & 1 & - & - & - & - \\
\hline V. splendidus II & 13 & 5 & 6 & 13 & 1 \\
\hline
\end{tabular}

\section{RESULTS}

\section{Larval survival and dominant bacteria}

High larval mortalities occurred in untreated groups in both experimental tanks (89\%) and regular production tanks $(72 \%)$, while in streptomycin-treated groups the cumulative mortality was 31 and $53 \%$, respectively, in experimental and regular tanks. In experimental tanks, larval mortality escalated on Day 5 , when dead and moribund larvae aggregated on the bottom of the untreated tank. Compared with actively swimming larvae, dead and moribund larvae had an about 1000 -fold higher bacterial load (Table 1). However, the difference in bacterial load between active and inactive larvae in the streptomycin-treated tank was not so marked. The number of presumptive vibrios was very high in inactive larvae in the untreated tank with $10^{3}$ cfu larva ${ }^{-1}$ (Table 1). Qualitative analysis of bacterial flora of both the study groups revealed a high proportion of vibrios in inactive larvae, while in the tank water vibrios were totally absent (Fig. 1a). A large number of the presumptive vibrios in all samples were identified as Vibrio splendidus II, and strains of $V$. harveyi were also found associated with infected larval samples from the untreated tank (Table 2).

In regular production tanks, increased rate of larval mortality was observed on Day 4, but the total bacterial load and presumptive vibrios in inactive larvae were lower than those in the experimental tanks maintained at higher larval density (Table 3). However, $95 \%$ of the isolates on MA from inactive larvae in the untreated tank were identified as Vibrio species (Fig. 1b). Vibrio was also the most dominant population from samples of active larvae $(65 \%)$ and water $(35 \%)$ in the untreated tank and inactive larvae (45\%) in the treated tank. V. splendidus II was detected from all samples examined, and was particularly dominant in inactive larvae (Table 4). $V$. harveyi was also detected in larvae of treated and untreated groups.

Most strikingly, all the samples of inactive larvae were found to contain a higher proportion of sucrose-negative vibrios (green-coloured colonies on TCBS) than sucrose-fermenting vibrios, constituting 72 to $90 \%$ of the total presumptive vibrios (Tables $1 \& 3$ ). Except in active larvae, where the 
(a)

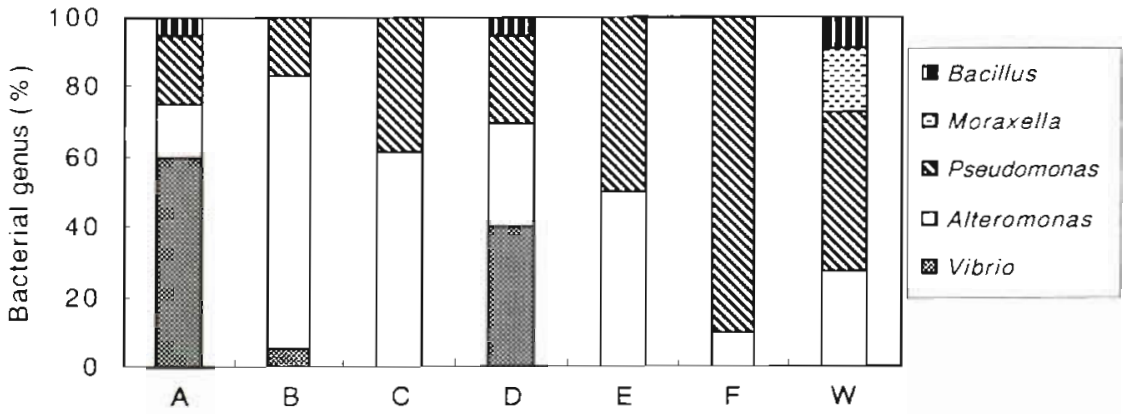

(b)

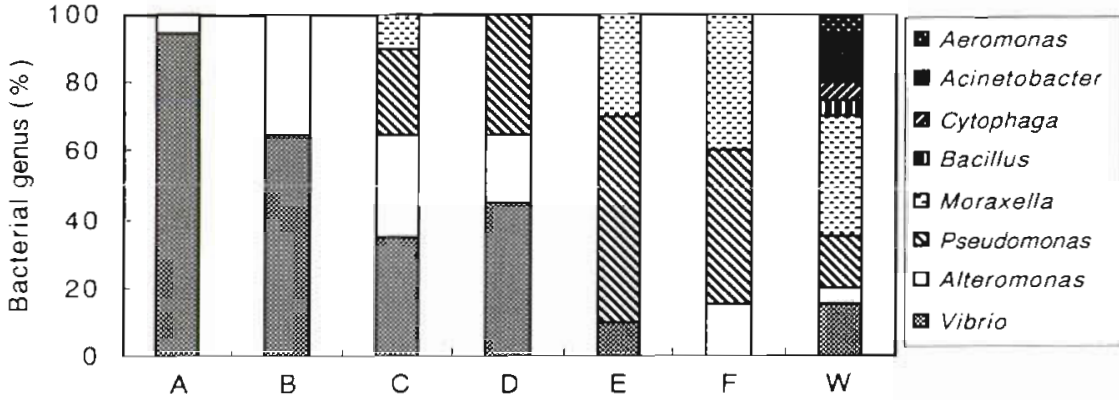

(c)

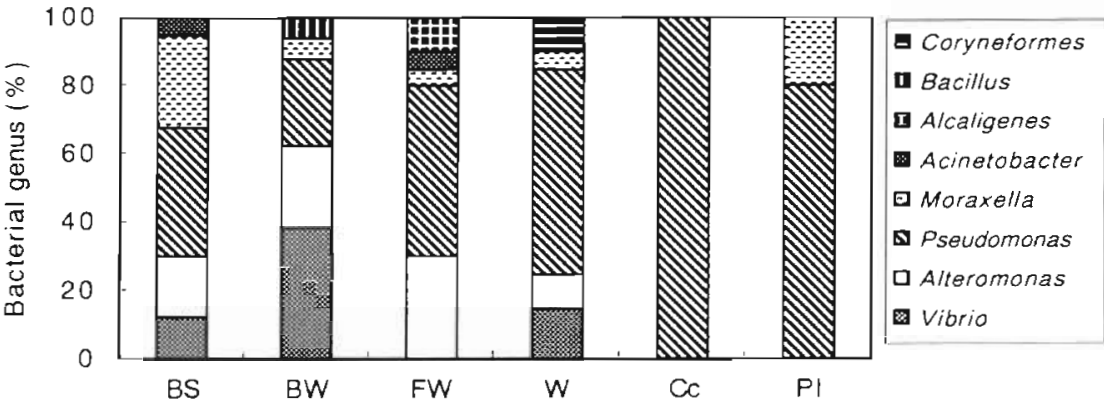

Fig. 1. Qualitative analysis of bacterial flora. (a) Samples from experimental tanks, (b) samples from regular production tanks, and (c) samples from other sources. A, B and C: inactive larvae, active larvae and water. respectively, from untreated tanks; $D, E$ and $F$ : inactive larvae, active larvae and water, respectively, from streptomycintreated tanks. W: raw seawater; BS: broodstock; BW: breeder tank water; FW: filtered seawater; Cc: Chaetoceros calcitrans; PI: Pavlova lutheri sucrose-positive vibrios outnumbered sucrose-negative vibrios, all other samples, including raw seawater, had a higher proportion of sucrose-negative vibrios. isolated from gonad samples of broodstock upon direct streaking on TCBS. In contrast, vibrios were not detected from the normal flora of algal cultures, which primarily consisted of Pseudomonas.

\section{Dominant bacteria in other samples}

Quantitative and qualitative analyses of samples of adult oysters, breeder tank water, feed consisting of 2 algal cultures (Chaetoceros calcitrans and Pavlova lutheri), raw seawater and $1 \mu \mathrm{m}$-filtered seawater demonstrated the presence of vibrio populations in broodstock, breeder tank water and raw seawater, with a high proportion of sucrose-negative vibrios (Table 5, Fig. 1c). Vibrio harveyi and $V$. splendidus II were dominant among vibrios of these 3 samples. Strains of $V$. splendidus II were also
Table 3. Quantitative bacterial analysis of samples from production tanks

\begin{tabular}{|c|c|c|c|c|}
\hline Sample & $\begin{array}{r}\text { Total viabl } \\
\text { (cfu g } \mathrm{g}^{-1} \text { or } \mathrm{ml}^{-1} \text { ) }\end{array}$ & $\begin{array}{l}\text { e count } \\
\text { (cfu larva-1) }\end{array}$ & $\begin{array}{l}\text { Presumptive vi } \\
\text { (cfu g }^{-1} \text { or } \mathrm{ml}^{-1} \text { ) }\end{array}$ & $\begin{array}{l}\text { brio count } \\
\text { (cfu larva }^{-1} \text {. }\end{array}$ \\
\hline \multicolumn{5}{|l|}{ Untreated tank } \\
\hline Inactive larvae & $7.2 \times 10^{8}$ & $5.7 \times 10^{2}$ & $9.9 \times 10^{7}(90)$ & $7.8 \times 10^{1}$ \\
\hline Active larvae & $6.4 \times 10^{6}$ & $5.0 \times 10^{0}$ & $1.3 \times 10^{h}(41)$ & $1.0 \times 10^{0}$ \\
\hline Tank water & $2.0 \times 10^{3}$ & & $4.0 \times 10^{1}(88)$ & \\
\hline \multicolumn{5}{|c|}{ Streptomycin-treated $\tan k$} \\
\hline Inactive larvae & $6.9 \times 10^{7}$ & $5.4 \times 10^{1}$ & $1.4 \times 10^{7}(75)$ & $1.1 \times 10^{1}$ \\
\hline Active larvae & $1.1 \times 10^{7}$ & $8.4 \times 10^{0}$ & $1.3 \times 10^{5}(39)$ & $0.1 \times 10^{0}$ \\
\hline Tank water & $1.2 \times 10^{4}$ & & $1.5 \times 10^{1}(50)$ & \\
\hline Raw seawater & $5.2 \times 10^{2}$ & & $1.0 \times 10^{1}(100)$ & \\
\hline
\end{tabular}


Table 4. Vibrio species associated with samples from regular production tanks, $\mathrm{n}=$ number of isolates picked for species characterization

\begin{tabular}{|c|c|c|c|c|c|c|c|}
\hline \multirow[t]{2}{*}{ Species } & \multicolumn{3}{|c|}{ Untreated tank } & \multicolumn{3}{|c|}{ Streptomycin-treated tank } & \multirow{2}{*}{$\begin{array}{c}\text { Raw } \\
\text { seawater } \\
(\mathrm{n}=3)\end{array}$} \\
\hline & $\begin{array}{c}\text { Inactive } \\
\text { larvae } \\
(\mathrm{n}=29)\end{array}$ & $\begin{array}{l}\text { Active } \\
\text { larvae } \\
(n=22)\end{array}$ & $\begin{array}{c}\text { Tank } \\
\text { water } \\
(n=10)\end{array}$ & $\begin{array}{l}\text { Inactive } \\
\text { larvae } \\
(\mathrm{n}=15)\end{array}$ & $\begin{array}{l}\text { Active } \\
\text { larvae } \\
(n=7)\end{array}$ & $\begin{array}{c}\text { Tank } \\
\text { water } \\
(n=2)\end{array}$ & \\
\hline V. destuarinus & - & 4 & - & - & 2 & - & - \\
\hline V.alginolyticus & 1 & - & - & - & 1 & - & - \\
\hline V. anguillarum-like & - & - & - & 3 & - & - & - \\
\hline V. harveyi & 4 & 2 & - & 2 & 1 & - & - \\
\hline V. mediterranei & - & 6 & -- & - & 1 & - & - \\
\hline$V$. pelagius I & - & - & - & - & - & - & 1 \\
\hline Vibrio Phenon 14 & - & - & 1 & - & - & 1 & - \\
\hline$V$. splendidus I & 3 & - & 2 & 2 & - & - & - \\
\hline V. splendidus II & 21 & 10 & 7 & 8 & 2 & 1 & 2 \\
\hline
\end{tabular}

Table 5. Quantitative bacteriological analysis of hatchery samples

\begin{tabular}{|c|c|c|}
\hline Sample & $\begin{array}{l}\text { Total viable } \\
\text { count } \\
\text { (cfu } \mathrm{ml}^{-1} \text { or } \mathrm{g}^{-1} \text { ) }\end{array}$ & $\begin{array}{c}\text { Presumptive vibrio } \\
\text { count } \\
\text { (cfu m- } \text { or }^{-1} \text { ) }\end{array}$ \\
\hline Breeder & $1.9 \times 10^{5}$ & $1.8 \times 10^{3}(60)$ \\
\hline Breeder tank water & $6.2 \times 10^{4}$ & $2.5 \times 10^{2}(69)$ \\
\hline Chaetoceros calcitrans & $5 \quad 3.0 \times 10^{10}$ & $<1.0 \times 10^{\prime}$ \\
\hline Pavlova lutheri & $1.2 \times 10^{10}$ & $<1.0 \times 10^{1}$ \\
\hline Raw seawater & $1.3 \times 10^{3}$ & $3.0 \times 10^{1}(83)$ \\
\hline Filtered seawater & $1.5 \times 10^{3}$ & $<1.0 \times 10^{1}$ \\
\hline
\end{tabular}

\section{Pathogenicity of Vibrio spp.}

The pathogenicity of the tested Vibrio spp. against oyster larvae is presented in Table 6 . Five of the tested strains of $V$. splendidus II (strain nos. 58, 59, 60, Q3 and 102) caused $100 \%$ or near $100 \%$ mortality at $10^{5} \mathrm{cfu}$ $\mathrm{ml}^{-1}$ and their $\mathrm{LD}_{50}$ values were equal to or less than $5.2 \times 10^{4} \mathrm{cfu} \mathrm{m}^{-1}$ None of the strains of $V$. harveyi caused remarkable mortalities, even at concentrations of $10^{7} \mathrm{cfu} \mathrm{ml}^{-1}$, nor did strains of $V$. anguillarum-like and $V$. alginolyticus. Strain-to-strain variations in pathogenicity were observed among $V$. splendidus II, and

Table 6. Pathogenicity of Vibrio species against oyster larvae

\begin{tabular}{|c|c|c|c|c|c|c|c|c|c|}
\hline \multirow{2}{*}{$\begin{array}{l}\text { Strain } \\
\text { no. }\end{array}$} & \multirow{2}{*}{ Species } & \multirow{2}{*}{ Source } & \multirow{2}{*}{$\begin{array}{c}\text { Coefficient } \\
\text { of bacterial } \\
\text { density }\end{array}$} & \multicolumn{5}{|c|}{ Larval survival at } & \multirow{2}{*}{$\begin{array}{c}\mathrm{LD}_{50} \\
\left(\mathrm{cfuml} \mathrm{ml}^{-1}\right)\end{array}$} \\
\hline & & & & $10^{6}$ & $10^{5}$ & $\begin{array}{c}10^{4} \\
\left(\mathrm{cfu} \mathrm{ml^{-1 }}\right)\end{array}$ & $10^{3}$ & Control & \\
\hline 31 & V. splendidus II & Breeder & $3.4 x$ & 89 & 83 & 90 & 98 & 99 & $>3.4 \times 10^{6}$ \\
\hline V11 & V. splendidus II & Active larvảe & $5.4 \times$ & 83 & 100 & 100 & 100 & 100 & $>5.4 \times 10^{6}$ \\
\hline J13 & V. splendidus II & Seawater & $13.5 x$ & 100 & 100 & 100 & 99 & 100 & $>1.3 \times 10^{7}$ \\
\hline 43 & V. splendidus II & Breeder tank & $3.1 \times$ & 96 & 100 & 100 & 100 & 100 & $>3.1 \times 10^{6}$ \\
\hline 72 & V. splendidus Il & Larval tank & $5.4 \times$ & 97 & 99 & 100 & 100 & 100 & $>5.4 \times 10^{6}$ \\
\hline 58 & V. splendidus II & Inactive larvae & $9.2 \times$ & 0 & 0 & 0 & 76 & 100 & $4.2 \times 10^{4}$ \\
\hline 59 & V. splendidus II & Inactive larvae & $6.9 \times$ & 0 & 1.5 & 26 & 96 & 97 & $2.9 \times 10^{4}$ \\
\hline 60 & V. splendidus II & Inactive larvae & $9.3 \times$ & 0 & 0 & 0 & 49 & 100 & $<9.3 \times 10^{3}$ \\
\hline 63 & V. splendidus II & Inactive larvae & $3.2 \times$ & 65 & 82 & 96 & 100 & 100 & $>3.2 \times 10^{6}$ \\
\hline Q1 & V. splendidus II & Inactive larvae & $1.0 \times$ & 82 & 93 & 94 & 99 & 100 & $>1.0 \times 10^{6}$ \\
\hline Q3 & V. splendidus ll & Inactive larvae & $10.6 x$ & 0 & 0 & 21 & 95 & 100 & $4.3 \times 10^{4}$ \\
\hline Q4 & V. splendidus II & Inactive larvae & $2.5 x$ & 96 & 95 & 98 & 98 & 98 & $>2.5 \times 10^{6}$ \\
\hline 102 & V. splendidus II & Inactive larvae & $2.2 \times$ & 0 & 4 & 79 & 96 & 99 & $5.2 \times 10^{4}$ \\
\hline 105 & V. splendidus II & Inactive larvae & $3.3 \times$ & 14 & 59 & 100 & 100 & 100 & $5.9 \times 10^{5}$ \\
\hline 36 & V. harveyi & Breeder & $17.5 x$ & 88 & 98 & 96 & 95 & 99 & $>1.8 \times 10^{7}$ \\
\hline N14 & V. harveyi & Breeder tank & $10.0 \times$ & 94 & 98 & 99 & 100 & 100 & $>1.0 \times 10^{7}$ \\
\hline 56 & V. harveyi & Inactive larvae & $18.7 \times$ & 93 & 92 & 95 & 100 & 100 & $>1.9 \times 10^{7}$ \\
\hline Q6 & V. harveyi & Inactive larvae & $18.2 \times$ & 72 & 88 & 95 & 100 & 100 & $>1.8 \times 10^{7}$ \\
\hline 76 & V. harveyi & Larval tank & $20.0 \times$ & 94 & 97 & 97 & 99 & 99 & $>2.0 \times 10^{7}$ \\
\hline B3 & V. harveyi & Active larvae & $6.9 x$ & 100 & 200 & 100 & 100 & 100 & $>6.9 \times 10^{6}$ \\
\hline 62 & V. anguillarum-like & Inactive larvae & $15.7 \times$ & 100 & 100 & 100 & 100 & 100 & $>1.6 \times 10^{7}$ \\
\hline O6 & $V$ anguillarum-like & Inactive larvae & $4.0 \times$ & 100 & 100 & 100 & 100 & 100 & $>4.0 \times 10^{6}$ \\
\hline 37 & $V$. alginolyticus & Breeder & $9.1 \times$ & 92 & 96 & 100 & 100 & 100 & $>9.1 \times 10^{6}$ \\
\hline
\end{tabular}


Table 7. Drug sensitivity of isolates of Vibrio splendidus II. $\mathrm{S}=$ sensitive; $\mathrm{WS}=$ weakly sensitive; $\mathrm{R}=$ resistant

\begin{tabular}{|c|c|c|c|c|c|c|c|c|c|c|c|c|c|c|c|}
\hline $\begin{array}{l}\text { Strain } \\
\text { no. }\end{array}$ & $S M$ & KM & VCM & $A B P C$ & MPIPC & EM & OTC & CEX & CPZ & CEZ & SBT-CPZ & $\mathrm{NA}$ & $\mathrm{CP}$ & $N B$ & $\mathrm{CL}$ \\
\hline 31 & $S$ & WS & $\mathrm{R}$ & S & WS & S & S & S & S & WS & S & S & S & WS & S \\
\hline 43 & WS & $\mathrm{S}$ & $\mathrm{R}$ & S & $\mathrm{R}$ & $S$ & S & S & S & $\mathrm{S}$ & S & $\mathrm{S}$ & S & $\mathrm{S}$ & S \\
\hline 58 & $S$ & WS & $\mathrm{R}$ & WS & $\mathrm{R}$ & S & $\mathrm{S}$ & WS & $\mathrm{R}$ & S & S & S & $S$ & S & S \\
\hline 59 & S & $\mathrm{S}$ & $\mathrm{R}$ & WS & R & S & S & WS & $\mathrm{R}$ & WS & $\mathrm{S}$ & S & S & S & S \\
\hline 60 & S & S & $\mathrm{R}$ & WS & R & $S$ & S & WS & $\mathrm{S}$ & WS & WS & $S$ & S & S & $\mathrm{S}$ \\
\hline 63 & S & S & $\mathrm{R}$ & WS & $\mathrm{R}$ & WS & S & WS & $\mathrm{S}$ & $\mathrm{R}$ & $\mathrm{S}$ & S & $S$ & WS & WS \\
\hline 72 & WS & S & $\mathrm{R}$ & WS & R & $\mathrm{S}$ & $S$ & WS & WS & WS & $\mathrm{S}$ & S & S & S & $\mathrm{S}$ \\
\hline Q1 & $\mathrm{S}$ & $\mathrm{S}$ & $R$ & WS & $\mathrm{R}$ & $S$ & $S$ & WS & $\mathrm{S}$ & WS & $\mathrm{S}$ & S & $S$ & S & $S$ \\
\hline Q4 & S & WS & $R$ & WS & $\mathrm{R}$ & S & $\mathrm{S}$ & WS & WS & WS & $\mathrm{S}$ & S & S & S & S \\
\hline $\mathrm{J} 13$ & WS & WS & $\mathrm{R}$ & $\mathrm{S}$ & WS & $\mathrm{S}$ & $\mathrm{S}$ & WS & $\mathrm{R}$ & $S$ & WS & $\mathrm{S}$ & $\mathrm{S}$ & $S$ & $\mathrm{~S}$ \\
\hline 102 & $\mathrm{~S}$ & $\mathrm{~S}$ & $\mathrm{R}$ & WS & R & $S$ & $\mathrm{~S}$ & WS & $\mathrm{S}$ & WS & WS & $\mathrm{S}$ & $\mathrm{S}$ & $S$ & $S$ \\
\hline 105 & $S$ & $\mathrm{~S}$ & $\mathrm{R}$ & S & R & S & $\mathrm{S}$ & WS & S & $S$ & $\mathrm{~S}$ & $S$ & S & $S$ & $S$ \\
\hline E7 & S & WS & $\mathrm{R}$ & S & $R$ & S & $S$ & WS & WS & S & WS & S & S & S & $S$ \\
\hline \multirow{5}{*}{\multicolumn{4}{|c|}{$\begin{array}{l}\mathrm{SM}=\text { Streptomycin }(50 \mu \mathrm{g}) \\
\mathrm{KM}=\text { Kanamycin }(50 \mu \mathrm{g}) \\
\mathrm{VCM}=\text { Vancomycin }(5 \mu \mathrm{g}) \\
\mathrm{ABPC}=\text { Ampicillin }(30 \mu \mathrm{g}) \\
\mathrm{MPIPC}=\text { Oxycillin }(30 \mu \mathrm{g})\end{array}$}} & & \multirow{5}{*}{\multicolumn{5}{|c|}{$\begin{array}{l}\text { EM }=\text { Erythromycin }(30 \mu \mathrm{g}) \\
\text { OTC }=\text { Oxytetracycline }(30 \mu \mathrm{g}) \\
\text { CEX }=\text { Cephalexin }(30 \mu \mathrm{g}) \\
\text { CPZ }=\text { Cefaperazolin }(30 \mu \mathrm{g}) \\
\text { CEZ }=\text { Cefazolin }(30 \mu \mathrm{g})\end{array}$}} & \multirow{5}{*}{\multicolumn{6}{|c|}{$\begin{array}{l}\text { SBT-CPZ = Sulfactum-cefaperaz } \\
\mathrm{NA}=\text { Nalidixic acid }(50 \mu \mathrm{g}) \\
\mathrm{CP}=\text { Chloramphenicol }(100 \mu \mathrm{g}) \\
\mathrm{NB}=\text { Novobiocin }(20 \mu \mathrm{g}) \\
\mathrm{CL}=\text { Colistin }(150 \mu \mathrm{g})\end{array}$}} \\
\hline & & & & & & & & & & & & & & & \\
\hline & & & & & & & & & & & & & & & \\
\hline & & & & & & & & & & & & & & & \\
\hline & & & & & & & & & & & & & & & \\
\hline
\end{tabular}

generally isolates from infected larvae were more virulent than those from other sources. Even among the strains of $V$. splendidus II isolated from infected larval samples, $\mathrm{LD}_{50}$ for 5 - to 8 -d-old larvae ranged from less than $9.3 \times 10^{3}$ (strain no. 60 ) to greater than $2.5 \times 10^{6} \mathrm{cfu}$ $\mathrm{ml}^{-1}$ (strain no. Q4). Inoculated bacteria could be reisolated from moribund/dead larvae in all experimental infections and larvae maintained in control wells were free of $V$. splendidus II. Signs of the disease in all challenge experiments were similar to those of natural infections with intense bacterial swarming around the shell margin, subsequent loss of cilia and velum followed by death of larvae. In some cases, dead larvae were observed with an intact velum.

Drug sensitivity patterns of the isolates of Vibrio splendidus II demonstrated that all the 13 isolates examined were sensitive to the majority of the drugs tested including streptomycin (Table 7). However, the isolates were resistant to vancomycin (peptide group) and oxycillin (penicillin group) and most of them were weakly sensitive to ampicillin (penicillin group) and cephalexin (cephem group).

\section{DISCUSSION}

Use of antibiotics for controlling the bacterial numbers in rearing water and improving the survival of bivalve larvae has been reported previously (Walne 1958, Tubiash et al. 1965, Brown \& Losee 1978, DiSalvo et al. 1978, Jeffries 1982, Nicolas et al. 1996). In the present study, the effect of streptomycin was clearly demonstrated by an increased larval survival in the antibiotic-treated group in both experimental and regular production tanks. Quantitative and qualitative analyses of bacterial flora associated with Crassostrea gigas larvae showed a high incidence of Vibrio, which was distinctly higher in diseased (dead/moribund) larvae reared without streptomycin treatment. Although these results gave a strong indication of the involvement of Vibrio in the disease, the presence of several species of Vibrio made it difficult to incriminate a particular causative agent in the recurrent outbreaks.

Pathogenicity assays conducted with different Vibrio isolates indicated V. splendidus biovar II as the causative agent of the disease in Crassostrea gigas larvae. $V$. alginolyticus, although reported to be pathogenic to oyster larvae (Tubiash et al. 1965, Jeffries 1982), did not produce larval mortalities in the present study, perhaps due to strain-to-strain variation. Interestingly, not all tested strains of $V$. splendidus II were virulent and most virulent strains were isolated from diseased larvae. Strains from sources other than diseased larvae were either less virulent or avirulent. Further detailed investigations are required to establish any clear relationship between the virulence of the cultures and their source of isolation. Virulent strains of $V$. splendidus II were pathogenic to $C$. gigas larvae at concentrations of $10^{4} \mathrm{cfu} \mathrm{ml}^{-1}$ in rearing water and at higher concentrations the mortality rate was more drastic. These high rates of mortality could also be due to the high water temperature $\left(25^{\circ} \mathrm{C}\right)$ maintained in all infection experiments, which favours rapid multiplication of bacteria, as observed by Riquelme et al. (1995a) in 
infection experiments with scallop larvae. The virulent isolates of $V$. splendidus II in this study were equally pathogenic as those of $V$. tubiashii, which is reported to be a primary pathogen on oyster larvae (Jeffries 1982), and as that of an unidentified Vibrio sp. against cockle Fulvia mutica larvae (Fujiwara et al. 1993).

The progressive signs of the disease included an abnormal circular pattern of swimming of larvae, bacterial swarming around the velum and shell margin detachment of cilia and velum and eventual death of larvae similar to earlier descriptions (Tubiash et al 1965, Brown \& Losee 1978, Elston \& Leibovitz 1980 Jeffries 1982, Lodeiros et al. 1987). Therefore, it is concluded that this disease was another case of bacillary necrosis caused by Vibrio splendidus biovar II. In all previous cases of bacillary necrosis, sucrose-positive vibrios have been reported to be involved, and Jeffries (1982) suggested that controlling the concentration of sucrose-fermenting vibrios (yellow colonies on TCBS) below $100 \mathrm{ml}^{-1}$ in rearing water could minimize losses attributable to vibriosis. However, the case reported here is quite different and sucrose-negative vibrios were dominant in all infected larval samples and in most other samples. Involvement of $V$. splendidus as an opportunistic pathogen in scallop larvae has recently been considered (Nicolas et al. 1996). However, the species was not detected in all outbreaks of disease and an unidentified Vibrio species was concluded to be the major pathogen of scallop larvae.

Sources of possible bacterial infections of bivalve larval cultures in hatcheries are generally the broodstock, algal feed and raw seawater (Elston 1984). During the present investigations, vibrios were not detected from algal cultures fed to both larvae and broodstock, making algal feed unlikely to be the source of infection. Strains of Vibrio splendidus II were detected in raw seawater as well as in broodstock. Detection of $V$. splendidus II strains in the gonad of adult oysters suggests the probability that broodstock could be the source and route of transmission of this pathogen, as already pointed out for Ostrea edulis (Lodeiros et al. 1987) and Argopecten purpuratus (Riquelme et al. 1995b). In the hatchery, unlike the larvae which are reared in $1 \mu \mathrm{m}$-filtered seawater, the broodstock are maintained and conditioned only in sand-filtered seawater before spawing. Therefore, there is a possibility that the strains of $V$. splendidus II entering the system from raw seawater could build up along with normal flora in adult oysters. When passed on to larvae, these strains might proliferate in stressed individuals and eventually infect healthy individuals, thus bringing about mass mortality in crowded culture systems. When treated with streptomycin, this pathogen is under control and mortality rates are low. The mechanism of infection may be a combination of invasive and toxic pathways as suggested in earlier studies (Elston \& Leibovitz 1980, Lodeiros et al. 1987. Nicolas et al. 1996). The involvement of toxins in the pathogenicity of $V$. splendidus II, although implicated in our preliminary studies, requires further investigation.

At present, there is no evidence of the emergence of streptomycin-resistant strains among the Vibrio splendidus II isolated in this study, although streptomycin has been used experimentally in the hatchery since 1995, and this treatment has been effective in preventing disease. Development of alternative methods to control the disease becomes imperative as prolonged use of antibiotics might result in the selection of antibiotic-resistant bacteria. Biological control of this pathogen and/or other effective means of preventing the build-up of Vibrio spp. by better management practices are necessary in the hatchery system.

\section{LITERATURE CITED}

Akashige S, Kusuki Y (1996) Artificial induction and larval survival of trıploids in the oyster Crassostrea gigas. Bull Hiroshima Fish Exp Stn 19:1-20

Alsina M. Blanch AR (1994a) A set of keys for biochemical identification of environmental Vibrio species. J Appl Bacteriol 76:79-85

Alsina M, Blanch AR (1994b) Improvement and update of a set of keys for biochemical identification of Vibriospecies. J Appl Bacteriol 77:719-721

Barry AL, Thornsberry C (1991) Susceptibility tests: diffusion test procedures. In: Balows A, Hauster WJ Jr, Herrmann $K L$, Isenberg HD, Shadomy HJ (eds) Manual of clinical microbiology. American Society of Microbiology, Washington, DC, p 1117-1125

Brown C, Losee E (1978) Observations on natural and induced epizootics of vibriosis in Crassostrea virginica larvae. J Invertebr Pathol 31:41-47

DiSalvo LH, Blecka J, Zebal R (1978) Vibrio anguillarum and larval mortality in a Calıtornia coastal shellfish hatchery. Appl Environ Microbiol 35:219-221

Elston RA (1984) Prevention and management of infectious diseases in intensive mollusc husbandry. J World Maricult Soc 15:284-300

Elston RA (1993) Infectious diseases of the Pacific oyster, Crassostrea gigas. Annu Rev Fish Dis 3:259-276

Elston R, Leibovitz L (1980) Pathogenesis of experimental vibriosis in larval American oysters, Crassostrea virginica. Can J Fish Aquat Sci 37:964-978

Elston R, Leibovitz L, Relyea D, Zatila J (1981) Diagnosis of vibriosis in a commercial oyster hatchery epizootic: diagnostic tools and mangement features. Aquaculture 24: $53-62$

Fujiwara M, Uyeno Y, Iwao A (1993) A Vibrio sp. associated with mortalities in cockle larvae Fulvia mutica (Mollusca: Cardiidae). Fish Pathol 28: 83-89

Hada HS, West PA, Lee JV, Stemmler J, Colwell RR (1984) Vibrio tubiashii sp. nov., a pathogen of bivalve mollusks. Int J Syst Bacteriol 34:1-4

Jeffries VE (1982) Three Vibrio strains pathogenic to larvae of Crassostrea gigas and Ostrea edulis. Aquaculture 29 $201-226$ 
Lodeiros C, Bolinches J, Dopazo CP, Toranzo AE (1987) Bacillary necrosis in hatcheries of Ostrea edulis in Spain. Aquaculture 65:15-29

Nicolas JL, Corre S, Gauthier G, Robert R, Ansquer D (1996) Bacterial problems associated with scallop Pecten maximus larval culture. Dis Aquat Org 27:67-76

Oceanographical Society of Japan (1990) Investigations of coastal environment, Manual II. Water quality and microorganisms. Koseisha Koseikaku Co Ltd, Tokyo, p 357-372

Reed LJ, Meunch H (1938) A simple method of estimating fifty per cent end points. Am J Hyg 27:493-497

Riquelme C, Hayashida G, Toranzo AE, Vilches J, Chavez P (1995a) Pathogenicity studies on a Vibrio anguillarumrelated (VAR) strain causing an epizootic in Argopecten

Editorial responsibility: Albert Sparks,

Seattle, Washington, USA purpuratus larvae cultured in Chile. Dis Aquat Org 22: $135-141$

Riquelme C, Hayashida $G$, Vergara $N$, Vasquez A, Morales $Y$, Chavez P (1995h) Bacteriology of the scallop Argopecten purpuratus (Lamarck, 1819) cultured in Chile. Aquaculture 138:49-60

Tubiash HS, Chanley PE, Leifson E (1965) Bacillary necrosis, a disease of larval and juvenile bivalve mollusks. I. Etiology and epizootiology. J Bacteriol 90:1036-1044

Tubiash HS, Colwell RR, Sakazaki R (1970) Marine vibrios associated with bacillary necrosis, a disease of larval and juvenile bivalve mollusks. J Bacteriol 103:271-272

Walne PR (1958) The importance of bacteria in laboratory experiments on rearing the larvae of Ostrea edulis (L.) J Mar Biol Assoc UK 37:415-425

Submitted: December 20, 1997; Accepted: March 21, 1998

Proofs received from author(s): June 10, 1998 